\title{
Performance Modeling of Fresnel-Based CPV Systems: Effects of Deformations under Real Operation Conditions
}

\author{
A.Cvetkovic ${ }^{1}$, R.Mohedano ${ }^{1}$, O.Gonzalez ${ }^{2}$, P.Zamora $^{3}$, P.Benitez ${ }^{1,3}$, \\ P.M.Fernandez ${ }^{2}$, A. Ibarreche ${ }^{2}$, M.Hernandez ${ }^{1}$, J.Chaves ${ }^{1}$, J.C.Miñano ${ }^{1,3}$ \\ ${ }^{1}$ LPI-Europe, Campus de Montegancedo, edificio Cedint, 28223 Pozuelo de Alarcón, Spain \\ ${ }^{2}$ Guascor Foton, Políg. Ind. Granada, parcela $i-48530$ Ortuella Spain \\ ${ }^{3}$ CEDINT, Universidad Politecnica de Madrid, Campus de Montegancedo, Edificio Cedint, 28223 Pozuelo de \\ Alarcón, Spain
}

\begin{abstract}
Getting a lower energy cost has always been a challenge for concentrated photovoltaic. The FK concentrator enhances the performance (efficiency, acceptance angle and manufacturing tolerances) of the conventional CPV system based on a Fresnel primary stage and a secondary lens, while keeping its simplicity and potentially low-cost manufacturing. At the same time F-XTP (Fresnel lens+reflective prism), at the first glance has better cost potential but significantly higher sensitivity to manufacturing errors. This work presents comparison of these two approaches applied to two main technologies of Fresnel lens production (PMMA and Silicone on Glass) and effect of standard deformations that occur under real operation conditions.
\end{abstract}

Keywords: concentrating optics, Fresnel lenses, PMMA, SOG, deformation analysis.

PACS: 42.79.Ek, 42.79.Bh, 88.40.F-, 88.40.fc

\section{INTRODUCTION}

A significant part of the high efficiency of multijunction solar cells may be lost during the manufacturing and assembling of a CPV system due to the low system tolerances. This loss can be avoided by making the manufacturing procedures more precise but this in general compromises the cost per watt (USD/W) of the CPV system. In order to achieve competitive system costs in mass-production, it is essential that CPV concentrators incorporate sufficient manufacturing tolerances. As primary optics in CPV systems are frequently used Fresnel lenses. During the operation these lenses are exposed to the variation of environmental conditions that can cause the deformation of lenses that compromises the system performance, and, again, the ratio USD/W. This paper presents the influence of common deformations in different technologies (PMMA and Silicone on glass) on the performance of conventional Fresnel-based CPV systems, compared to an advanced system, the Fresnel-Köhler (FK). [1][6]

The two systems that will be compared in this paper are: a Fresnel lens with reflexive truncated prism (F-XTP), and a LPI-FK approach. Optical designs have been developed by LPI, and F-XTP is commercialized by Guascor Foton Company.

Both cases will be analyzed under the typical deformations of two different technologies, PMMA and silicon-on-glass (SOG). The advantage of PMMA is its low weight, while the SOG is more resistant to scratching and erosions. Both of them tend to show deformations which are of different type: in the case of PMMA lenses, a shape warp (probably linked to moisture absorption of the acrylic) provokes the shift of the lens focus, while in the case of SOG the problem is a lens facets deformation due to the different thermal expansion of substrate and glass [2]. Additionally, the different thermal expansion of Fresnel lens parquets and housing causes displacements of the receiver (cell with secondary optics) with respect to the primary optics. All these thermal effects are modeled using finite element software, and their impact on performance analyzed through ray tracing and electrical modeling of the deformed CPV system. The analysis will determine the actual performance drops these effects cause on the case study systems. 

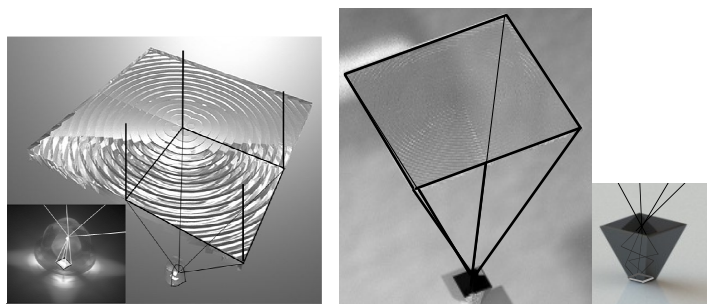

FIGURE 1. FK(left) and F-XTP (right) model

\section{PERFORMANCES}

All four models that will be studied are designed for the same concentration and the same cell size. However, different f-numbers (as defined to be the ratio of the distance between the cell and Fresnel lens to the Fresnel lens diagonal) have been studied since FK system performs well even when design is more compact. Find in the Table 1 design parameters.

TABLE 1. Design parameters

\begin{tabular}{lll}
\hline $\begin{array}{l}\text { Geometrical } \\
\text { concentration }\end{array}$ & $\begin{array}{l}\text { F+XTP } \\
700 \mathrm{x}\end{array}$ & $\begin{array}{l}\text { FK } \\
\text { Cell side }\end{array}$ \\
$\begin{array}{l}\text { Entry aperture } \\
\left(\mathrm{mm}^{2}\right)\end{array}$ & $\begin{array}{l}8.7 \mathrm{~mm} \\
230 \times 230\end{array}$ & $8.7 \mathrm{~mm}$ \\
$f \#$ & $230 \times 230$ \\
System height & 1.4 & 1.2 \\
\hline
\end{tabular}

Concentrator designs and their ray-tracing simulations have been done with the following features: (i) Fresnel lens: a) SOG $(n \approx 1.41)$ and b) PMMA $(n \approx 1.49)$, facet draft angle $=2^{\circ}$, vertex radius $=3$ $\mu \mathrm{m}$, facet height $<250 \mu \mathrm{m}$; (ii) SOE: a) FK: made of solar glass $(n \approx 1.52)$ coupled to the cell with a transparent silicone rubber of $\mathrm{n} \approx 1.41$ (e.g., Sylgard 182 of Dow Corning); b) XTP: made of high reflectivity mirror (iii) high efficiency $(\approx 38 \%)$ commercial triple-junction cell (in the case of XTP solar cell has been protected with thin silicon layer of $\mathrm{n} \approx 1.41$ ). Absorption in dielectric materials, Fresnel reflections and spectral and angular response of mirror are considered, but surface scattering is neglected. For the optical efficiency calculations have been considered all spectral transmissions of materials. Currents have been calculated for the AM1.5d ASMT G173 spectrum taking into account the EQE of the sub-cells and always calculating the limiting one.

Another useful merit function for a CPV optic is the concentration-acceptance product [1], which we define as:

$C A P=\sqrt{C_{g}} \sin \alpha$ where $\mathrm{Cg}$ is the geometric concentration and $\alpha$ the acceptance angle, defined as the incidence angle at which the concentrator collects $90 \%$ of the on-axis power. It is remarkable that for a given concentrator architecture, the CAP is rather constant with $\mathrm{Cg}$.

In the Table 2 is shown the result summary for all four designs. It can be noted that the F-XTP designs have higher efficiency for normal incidence (perfect tracking). That occurs because at perfect tracking only few rays get reflected in the prism. Considering the rest of relevant parameters FK is mainly superior. Higher acceptance angle is necessary to compensate tracking errors and good irradiance uniformity is necessary to avoid fill factor losses that can be produced due to the local current mismatch between the top and middle junction in solar cell. [3][4]

TABLE 2. Nominal design values for all analyzed cases

\begin{tabular}{lllll}
\multicolumn{5}{c}{ TABLE 2. Nominal design values for all analyzed cases } \\
\hline & F+XTP & F+XTP & FK & FK \\
PMMA & SOG & PMMA & SOG \\
Efficiency & $87.16 \%$ & $87.69 \%$ & $84.91 \%$ & $85.68 \%$ \\
$\begin{array}{l}\text { Isc } \\
\left(@ 900 \mathrm{~W} / \mathrm{m}^{2}\right)\end{array}$ & $5.75 \mathrm{~A}$ & $5.74 \mathrm{~A}$ & $5.51 \mathrm{~A}$ & $5.64 \mathrm{~A}$ \\
$\begin{array}{l}\text { Acceptance } \\
\text { angle }\end{array}$ & $0.81^{\circ}$ & $0.75^{\circ}$ & $1.15^{\circ}$ & $1.104^{\circ}$ \\
$\begin{array}{l}\text { Peak } \\
\text { irradiance }\end{array}$ & $1830 \mathrm{x}$ & $1780 \mathrm{x}$ & $690 \mathrm{x}$ & $730 \mathrm{x}$ \\
CAP & 0.37 & 0.38 & 0.53 & 0.51 \\
\hline
\end{tabular}

\section{Manufacturing tolerances}

Broadly speaking, the acceptance angle describes how some optics performs angularly. For instance, for a perfectly manufactured CPV system, it tells the maximum tracking error allowable if we want to assure a $90 \%$ of the maximum achievable power output (which occurs at normal incidence, perfect sunaim).

However, these values do not fully describe how sensitive is the system to manufacturing errors, such as misalignment between optical parts or between optical parts and receiver. Indeed, although such sensitivity is partly linked to the acceptance characteristic, the manufacturing tolerances cannot be deduced from the latter right away. Moreover, depending on the optical approach (geometry, number and type -mirrors, lenses, total internal reflection TIR- of surfaces...) a concentrator might be less sensitive to manufacturing inaccuracies even having lower acceptance values. The reasons behind the actual sensitivity of each system are not so clear in other cases, and the actual tolerances should be analyzed through ray tracing.

A simple tolerance analysis defining maximum shifts allowable in three directions has been carried out, fixing minimum acceptance and/or efficiency levels, and analyzing which one limits in each case. The study includes: 
- Lateral shifts (perpendicular to the optical axis Z, and parallel to the $\mathrm{X}$ and $\mathrm{Y}$ axes) of receivers (SOE+cell) with respect to the POE Fresnel lens.

- Longitudinal shifts (along the optical axis) of receivers both away from/towards the POE Fresnel lens.
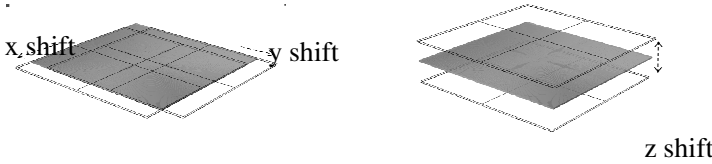

FIGURE 2. This work analyzes the effect of lateral and longitudinal shifts between the optical parts of the FK system

The following table shows the maximum shifts the concentrators can bear when we fix different acceptability criteria. The figures are referred to the cell size $l$ or the lens equivalent focal distance $f$ and have been calculated with an equivalent f-number (system optical depth f/lens diagonal; 1.2 for FK and 1.4 for XTP) and $\mathrm{Cg}=700 \times$. Criteria used were allowed movement to maintain the acceptance above $0.7^{\circ}$ (approximately $10 \%$ drop of nominal value for FXTP models). Can be noted that FK models even being more compact have two to four times higher tolerances. In the case of lateral alignment error can be permitted $3.5 \mathrm{~mm}$ misalignment for FK SOG and only $0.73 \mathrm{~mm}$ for F-XTP SOG model.

TABLE 3. Tolerances (criteria used was acceptance down to $0.7^{\circ}$, efficiency maintain unaffected)

\begin{tabular}{cll}
\hline \multicolumn{1}{c}{ Tolerances } & F+XTP & FK PMMA \\
& PMMA & \\
Lateral & $0.084 l$ & $0.41 l$ \\
Vertical & $0.0228 f$ & $0.0445 f$ \\
\hline \multicolumn{1}{c}{ Tolerances } & F+XTP SOG & FK SOG \\
Lateral & $0.084 l$ & $0.35 l$ \\
Vertical & $0.0134 f$ & $0.0331 f$ \\
\hline
\end{tabular}

As was already mentioned, color separation is very important in multijunction solar cells and can produce current mismatch that can lead to efficiency losses up to $5 \%$. [3][4] In the case of FK perfect color mixing is happening inside of tolerance range, while in the case of F-XTP can be noted color separation even in the nominal position. Further if moving SOE along $\mathrm{z}$-axis (vertical misalignment) can be noted separation between colors in the irradiance profiles that are different for top and for middle cells. In the case of FK this is not an issue (Figure 3).

\section{Influence of warp in PMMA models}

PMMA Fresnel lenses are low-weight, efficient and potentially inexpensive (continuous roll embossing produce large area array of lenses facilitating assembling and alignment). Although this technology seemed free of competitors for some time, the lens warp noticed (typically shows a equivalent curvature radius of $10 \mathrm{~m}$, is encouraging some manufacturers to try alternatives such as those based on SOG, since such warp implies important light leakage in systems of scarce acceptance.

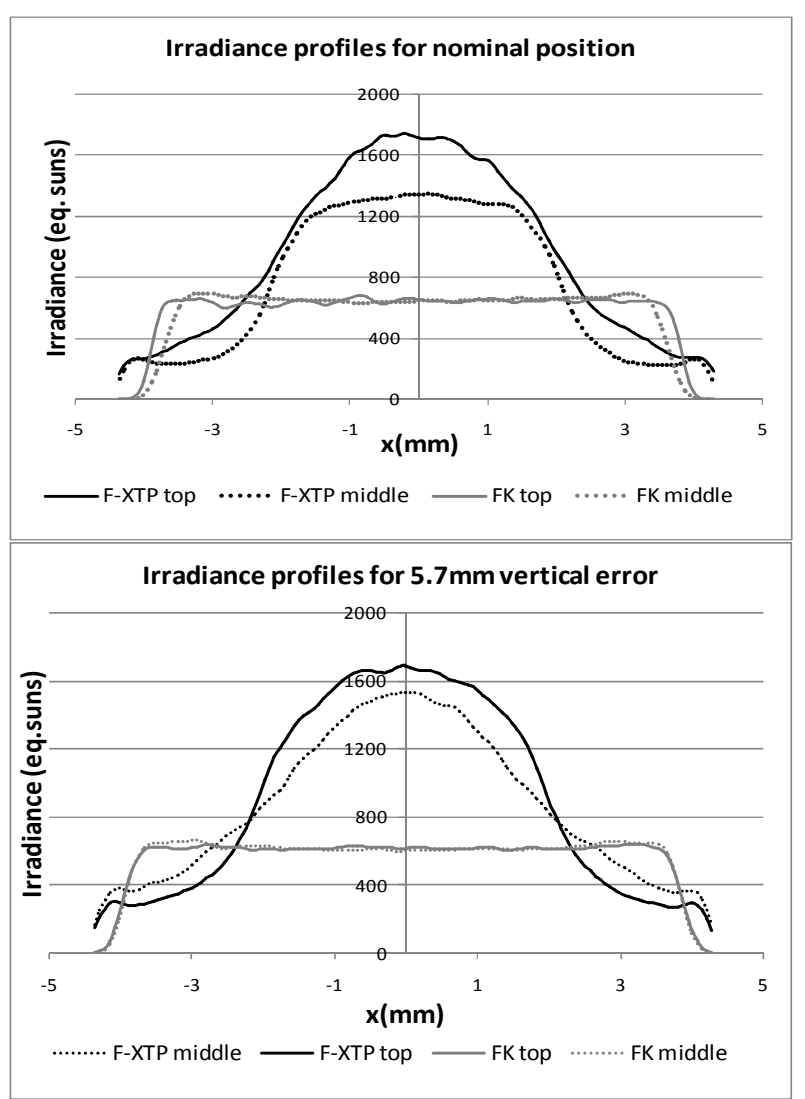

FIGURE 3. Irradiance profiles for both designs for two subcells; up: for nominal position; down: for $5.7 \mathrm{~mm}$ vertical displacement of SOE and cell

We have analyzed the effect of warp on performance, and found out that $\mathrm{FK}$ is quite insensitive to such, while in the case of F-XTP we lose about 7\% of acceptance, as Table 4 shows.

TABLE 4. Influence of PMMA Frensel lens warp

\begin{tabular}{lll}
\hline & F+XTP warp & FK warp \\
Efficiency & $86.51 \%$ & $82.29 \%$ \\
Isc & $5.65 \mathrm{~A}$ & $5.42 \mathrm{~A}$ \\
Acceptance angle & $0.76^{\circ}$ & $1.12^{\circ}$ \\
\hline
\end{tabular}




\section{Temperature deformations of SOG models}

SOG Fresnel lens have broad UV stable optical properties, abrasion and impact resistant exterior surface, and excellent dimensional stability over temperature and humidity.

A finite element model of the optical system of the figure has been performed in order to estimate the deformation and stress fields of the lens. Due to the complexity of the geometry, with a huge number of small teeth, a solid 3D model is not convenient and an axisymmetric model is created instead, that has been shown more suitable than flat deformation model. By following this strategy, the results have demonstrated to be accurate except for the corner regions as it can be understood. For the numerical calculation, a uniform temperature increment is applied to the lens. The lens working temperature is calculated from the thermal equilibrium of the system taking into account the absorbed solar energy and the heat dissipation mechanisms. The materials are considered to be elastic and linear and the system is supposed to be simply supported due to the low stiffness of the joint to the supporting structure.

As the silicon has a much larger thermal expansion coefficient than the glass, and a much smaller stiffness, the thin silicon layer develops low harmless compressive stresses. Silicone rubber is fixed to the glass and thus can't expand freely in horizontal direction while in the vertical direction has a complete freedom of expansion. This leads to the deformation of the Fresnel prisms that get steeper slope angles with higher temperature, what causes an increase of the size of the focal spot, and at the same time tends to decrease the change in focal length. In a CPV module a change of these to parameters might decrease optical efficiency by spilling light off the active solar cell area (in the case no secondary optics is used). [2]

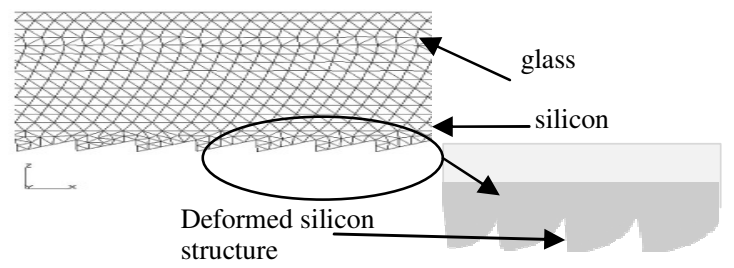

FIGURE 4. Finite element method simulation and schematic presentation of thermal deformation of SOG Fresnel lens when temperature is increased (deformations are strongly exaggerated)

Since both considered cases work with secondary optics, the effect of deformations in the case of perfect alignment (normal incidence) is not noticeable. In the case of XTP design can be noticed decrement of efficiency due to more rays that suffer reflection on the prism. In the case of FK can be noted that is highly tolerant, and almost no change of efficiency is noted for the temperature of $40^{\circ}$ above the ambient $\left(\mathrm{T}_{\mathrm{amb}}=20^{\circ} \mathrm{C}\right)$. This can be explained by the fact that draft angle on the teeth of Fresnel lens is lower what compensate losses caused by deformation of the focal spot. On the graphic in the Figure 5 can be seen the variation of $I_{\mathrm{SC}}$ and $\alpha$ with lens temperature. FK is insensitive for all studied temperature deformation (up to $80^{\circ} \mathrm{C}$ ) while in the case of F-XTP can be noted significant decrement of efficiency and Isc above $60^{\circ} \mathrm{C}$. This can be explained with higher vertical tolerances in the case of FK that are consequence of higher half acceptance angle.

\section{CONCLUSIONS}

Both presented design are technologically simple Fresnel based systems that are have good tolerances. F-XTP has higher nominal efficiency, but FK outstands in acceptance and tolerance, what can be significant at the array level. Apart from attaining medium-high concentration-acceptance angle product (CAP) FK is capable of providing perfect irradiance uniformity on the solar cell, which is one of the factors to make a system durable. Considering different technologies, both systems perform similar with PMMA and SOG Fresnel lenses. While in the case of F-XTP almost no difference between two technologies is noted (similar sensitivity, efficiency and acceptance), in the case of FK, with PMMA POE has better efficiency, and slightly higher tolerances.

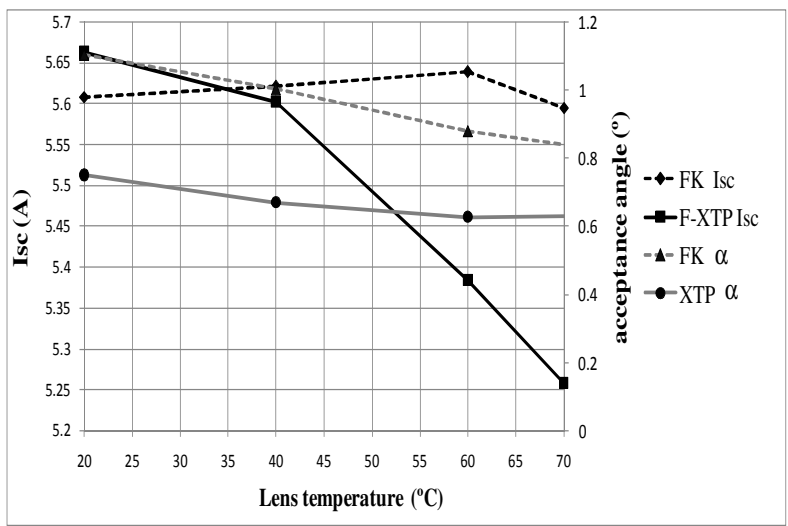

FIGURE 5. Variation of efficiency and acceptance angle with temperature of the Fresnel lens for both cases, XTP solid and FK dashed line

Considering that both models at the unit level have measured DC efficiency above 30\% (Guascor F-XTP even et the module level reach $31.5 \%,[5][7])$ and that FK model is less sensitive to deformations and manufacturing errors this advantage can be used to lower the cost of system and with it decrease the cost of energy. 


\section{ACKNOWLEDGMENTS}

Support for this work partly comes from the EC and Spanish Ministry MICINN under projects SSL4EU (Grant agreement n ${ }^{\circ} 257550$, FP7/20072013) and SIGMASOLES (PSE-440000-2009-8), respectively.

\section{REFERENCES}

1. P.Benítez et al (2010)"High performance Fresnel-based photovoltaic concentrator", Energy Express, OSA. Vol. 18, Issue S1, pp. A25-A40

2. J.Hornung et al, (2010). Temperature Dependent Measurements and Simulation of Fresnel Lenses for $\mathrm{CPV}$, 6th International Conference on Concentrating Photovoltaics (CPV-6), Freiburg, Germany

3. A. Braun et al, "Localized radiation effects on tunnel diode transitions in multi-junction concentrator solar cells", Solar Energy Materials and Solar Cells 93, 16921695, (2009).

4. Kurtz S., M.J. O’Neill, "Estimating and controlling chromatic aberration losses for two-junction, twoterminal devices in refractive concentrator systems", 25th PVSC; pp.361-367, (1996)

5. A.Cvetkovic et al, (2010) Characterization of FresnelKöhler concentrator, Proc. 25th European PVSolar Energy Conference and Exibition, Valencia, Spain

6. FK is protected by US patent47654-0032-00-US (445 256)

7. http://www.photon-international.com/newsletter/ do cument/27785.pdf 